Supplement of Ocean Sci., 11, 139-158, 2015

http://www.ocean-sci.net/11/139/2015/

doi:10.5194/os-11-139-2015-supplement

Ocean Science

(C) Author(s) 2015. CC Attribution 3.0 License.

(c) (1)

Supplement of

\title{
Using empirical orthogonal functions derived from remote-sensing reflectance for the prediction of phytoplankton pigment concentrations
}

\author{
A. Bracher et al.
}

Correspondence to: A. Bracher (astrid.bracher@awi.de) 


\section{Supplement: Additional tables}

Table 1. List of cruises from which HPLC surface pigment data sets were used for the field (upper panel) and the satellite-based (lower panel) data set, including database (DB) or citation (ref.), cruise name, number of pigment data matched by $5 \times 5$ collocation criterium $(\mathrm{N})$, investigator, date and region.

Pigment data matched with field reflectances data

\begin{tabular}{llllll}
\hline DB/ref. & Cruise & Dates & Investigator & Region & $\mathrm{N}$ \\
\hline Own data & ANTXXIV/4 & 25 Apr-6 May 08 & Bettina Taylor & $35^{\circ} \mathrm{N}-10^{\circ} \mathrm{S}, 42^{\circ}-23^{\circ} \mathrm{W}$ & 11 \\
Own data & ANTXXV/1 & 10-24 Nov 08 & Bettina Taylor & $35^{\circ} \mathrm{N}-9^{\circ} \mathrm{S}, 21^{\circ}-14^{\circ} \mathrm{W}$ & 22 \\
Own data & ANTXXVI/4 & 24 Apr-9 May 10 & Bettina Taylor & $35^{\circ} \mathrm{N}-9^{\circ} \mathrm{S}, 27^{\circ}-13^{\circ} \mathrm{W}$ & 20 \\
\hline
\end{tabular}

Pigment data match with satellite reflectances data

\begin{tabular}{llllll}
\hline DB/ref. & Cruise & Dates & Investigator & Region & $\mathrm{N}$ \\
\hline BODC & AMT 12 & 27-31 May 03 & Tim Jickells & $12^{\circ} \mathrm{N}-10^{\circ} \mathrm{S}, 35^{\circ}-14^{\circ} \mathrm{W}$ & 3 \\
BODC & AMT 14 & 12-23 May 04 & Patrick Holligans & $32^{\circ} \mathrm{N}-7^{\circ} \mathrm{S}, 36^{\circ}-25^{\circ} \mathrm{W}$ & 5 \\
BODC & AMT 15 & 4-12 Oct 04 & Andy Rees & $34^{\circ} \mathrm{N}-8^{\circ} \mathrm{S}, 25^{\circ}-17^{\circ} \mathrm{W}$ & 5 \\
BODC & AMT 16 & 7-12 Jun 05 & Patrick Holligans & $14^{\circ} \mathrm{N}-2^{\circ} \mathrm{S}, 32^{\circ}-25^{\circ} \mathrm{W}$ & 5 \\
Own data & ANTXXIII/1 & 26 Oct-8 Nov 05 & Rüdiger Röttgers & $39^{\circ} \mathrm{N}-10^{\circ} \mathrm{S}, 21^{\circ}-4^{\circ} \mathrm{W}$ & 6 \\
SEABASS & EGEE3 & 29 May-4 Jul 05 & A.Subramamanian & $6^{\circ}-10^{\circ} \mathrm{S}, 10^{\circ}-3^{\circ} \mathrm{E}$ & 13 \\
SEABASS & Amma-rb-06 & 25 Jun-3 Jul 06 & Norman Nelson & $14^{\circ} \mathrm{N}-5^{\circ} \mathrm{S}, 23^{\circ} \mathrm{W}$ & 3 \\
SEABASS & EGEE5 & 10-11 Jun 07 & A Subramamanian & $8^{\circ}-5^{\circ} \mathrm{N}, 6^{\circ}-2^{\circ} \mathrm{W}$ & 2 \\
Own data & ANTXXIV/1 & 1-15 Nov 07 & Bettina Taylor & $35^{\circ} \mathrm{N}-6^{\circ} \mathrm{S}, 22^{\circ}-2^{\circ} \mathrm{W}$ & 15 \\
BODC & D325 & 17 Nov-15 Dec 07 & Ruth Airs & $26^{\circ}-16^{\circ} \mathrm{N}, 25^{\circ}-22^{\circ} \mathrm{W}$ & 9 \\
LOV & BonusGoodH & 14-28 Feb 08 & Josephine Ras & $18^{\circ}-8^{\circ} \mathrm{N}, 42^{\circ}-33^{\circ} \mathrm{W}$ & 2 \\
BODC & AMT 18 & 12-26 Oct 08 & Victor Martinez & $33^{\circ} \mathrm{N}-10^{\circ} \mathrm{S}, 41^{\circ}-24^{\circ} \mathrm{W}$ & 8 \\
Own data & ANTXXIV/4 & 25 Apr-6 May 08 & Bettina Taylor & $35^{\circ} \mathrm{N}-10^{\circ} \mathrm{S}, 42^{\circ}-23^{\circ} \mathrm{W}$ & 13 \\
Own data & ANTXXV/1 & 10-24 Nov 08 & Bettina Taylor & $35^{\circ} \mathrm{N}-9^{\circ} \mathrm{S}, 21^{\circ}-14^{\circ} \mathrm{W}$ & 4 \\
BODC & AMT 19 & 22 Oct-10 Nov 09 & Victor Martinez & $33^{\circ} \mathrm{N}-9^{\circ} \mathrm{S}, 41^{\circ}-24^{\circ} \mathrm{W}$ & 28 \\
Own data & ANTXXVI/4 & 24 Apr-9 May 10 & Bettina Taylor & $35^{\circ} \mathrm{N}-9^{\circ} \mathrm{S}, 27^{\circ}-13^{\circ} \mathrm{W}$ & 6 \\
Own data & MSM18-3 & 30 Jun-18 Jul 11 & Bettina Taylor & $3^{\circ} \mathrm{N}-6^{\circ} \mathrm{S}, 21^{\circ} \mathrm{W}-0^{\circ}$ & 34 \\
\hline
\end{tabular}


Table 2. Specific pigments measured in samples collocated to the satellite-based $5 \times 5$ pixels (s) and field (f) reflectance data sets: Maximum (max), minimum (min), mean and standard deviation (stdv) in $\mathrm{mg} \mathrm{m}^{-3}$ (for PE relative units are given), number and percentage of samples where specific pigments had a concentration of $0 \mathrm{mg} \mathrm{m}^{-3}$ (nd and nd \%, respectively) or were not measured in most samples (nm). Prasino, Allo, Lut, $\beta$-caroten, TPhaeo and chlorophyllide- $a$ were only detected in less than $60 \%$ of the samples at both data sets and are not listed here. Full pigment names and abbreviations are given in chapter 2.3.1.

\begin{tabular}{llllllllll} 
Pigment & MVChla & DVChla & TChla & TChlb & Chl-c1/2 & Chl-c3 & Fuco & But & Hex \\
\hline max-s & 2.9920 & 0.1439 & 3.5529 & 0.1203 & 0.2607 & 0.1123 & 1.7931 & 0.1950 & 0.4752 \\
min-s & 0.0048 & 0.0052 & 0.0048 & 0.0014 & 0.0003 & 0.0001 & 0.0010 & 0.0013 & 0.0030 \\
mean-s & 0.4075 & 0.0501 & 0.4681 & 0.0318 & 0.0645 & 0.0177 & 0.0887 & 0.0455 & 0.1229 \\
stdv-s & 0.4677 & 0.0374 & 0.5214 & 0.0352 & 0.0630 & 0.0313 & 0.2596 & 0.0478 & 0.1253 \\
nd-s & 0 & 15 & 0 & 41 & 14 & 51 & 15 & 14 & 8 \\
nd-s [\%] & $0 \%$ & $9 \%$ & $0 \%$ & $24 \%$ & $8 \%$ & $30 \%$ & $9 \%$ & $8 \%$ & $5 \%$ \\
max-f & 3.9759 & 0.2356 & 4.1505 & 1.1164 & 1.1568 & 0.6877 & 1.7890 & 0.3455 & 1.5512 \\
min-f & 0.0228 & 0.0097 & 0.0326 & 0.0034 & 0.0022 & 0.0015 & 0.0017 & 0.0019 & 0.0074 \\
mean-f & 0.0408 & 0.0627 & 0.4745 & 0.0560 & 0.0689 & 0.0413 & 0.0913 & 0.0303 & 0.1137 \\
stdv-f & 0.8091 & 0.0509 & 0.8126 & 0.1641 & 0.1809 & 0.1025 & 0.2840 & 0.0529 & 0.2437 \\
nd-f & 0 & 4 & 0 & 18 & 0 & 1 & 1 & 0 & 0 \\
nd-f [\%] & $0 \%$ & $8 \%$ & $0 \%$ & $34 \%$ & $0 \%$ & $2 \%$ & $2 \%$ & $0 \%$ & $0 \%$ \\
\hline
\end{tabular}

\begin{tabular}{lllllllllll} 
Pigment & Peri & PSC & Diadino & Diato & Viola & Zea & Caro & PPC & PE & Neo \\
\hline max-s & 0.0585 & 1.8917 & 0.3825 & 0.0482 & 0.0235 & 0.4660 & 0.1909 & 0.5332 & $\mathrm{~nm}$ & $\mathrm{~nm}$ \\
min-s & 0.0005 & 0.0028 & 0.0001 & 0.0006 & 0.0001 & 0.0086 & 0.0014 & 0.0178 & $\mathrm{~nm}$ & $\mathrm{~nm}$ \\
mean-s & 0.0146 & 0.2787 & 0.0532 & 0.0078 & 0.0016 & 0.1214 & 0.0316 & 0.1567 & $\mathrm{~nm}$ & $\mathrm{~nm}$ \\
stdv-s & 0.0160 & 0.3267 & 0.0677 & 0.0116 & 0.0039 & 0.0868 & 0.0296 & 0.1069 & $\mathrm{~nm}$ & $\mathrm{~nm}$ \\
nd-s & 58 & 0 & 9 & 66 & 87 & 1 & 23 & 0 & $\mathrm{~nm}$ & $\mathrm{~nm}$ \\
nd-s [\%] & $35 \%$ & $0 \%$ & $5 \%$ & $39 \%$ & $52 \%$ & $1 \%$ & $14 \%$ & $0 \%$ & $\mathrm{~nm}$ & $\mathrm{~nm}$ \\
max-f & 0.0477 & 3.6995 & 0.6315 & 0.0797 & 0.2532 & 0.3526 & 0.2626 & 1.0107 & 0.7296 & 0.1417 \\
min-f & 0.0017 & 0.0092 & 0.0045 & 0.0008 & 0.0006 & 0.0225 & 0.0029 & 0.0302 & 0.0008 & 0.0013 \\
mean-f & 0.0051 & 0.2404 & 0.0517 & 0.0081 & 0.0104 & 0.0933 & 0.0311 & 0.2004 & 0.0983 & 0.0066 \\
stdv-f & 0.0084 & 0.5666 & 0.1046 & 0.0143 & 0.0371 & 0.534 & 0.0426 & 0.2233 & 0.1496 & 0.0222 \\
nd-f & 17 & 0 & 1 & 8 & 14 & 0 & 0 & 0 & 0 & 29 \\
nd-f[\%] & $32 \%$ & $0 \%$ & $2 \%$ & $15 \%$ & $26 \%$ & $0 \%$ & $0 \%$ & $0 \%$ & $0 \%$ & $55 \%$ \\
\hline & & & & & & & & & & \\
\hline
\end{tabular}


Table 3. Statistics of linear models using EOF modes based on full fit (MDPD, MPD, $P B, a$, $R M S E$, and $R^{2}$ ) and the mean values of validation over all permutations (MDPDcv, MPDcv, $R M S E c v$, and $R^{2} c v$ ) for all models established for all pigment and pigment groups and based on the six reflectance data sets: field data set with hyperspectral resolution (hyper_ $R_{R S}$ ) a) from 350 to $700 \mathrm{~nm}$ and b) from 380 to $700 \mathrm{~nm}$, c) field data set with multispectral (band_ $R_{R S}$ ) resolution and satellite data set with multispectral resolution (MERIS $\rho_{w N}$ data) using d) 1x1, e) $3 \times 3$, and f) $5 \times 5$ matchups.

a) Field data set hyperspectral resolution $350-700 \mathrm{~nm}$

\begin{tabular}{llrrrrrrrrrc} 
Pigment & $N$ & $M D P D$ & $M P D$ & \multicolumn{1}{c}{$P B$} & $a$ & $R M S E$ & $R^{2}$ & $M D P D_{c V}$ & $M P D_{c v}$ & $R M S E_{c v}$ & $R_{c v}^{2}$ \\
\hline TChla & 53 & 23 & 33 & 9 & -0.25 & 0.43 & 0.82 & 33 & 48 & 0.60 & 0.67 \\
TChlb & 53 & 85 & 251 & 195 & -1.62 & 1.61 & 0.70 & 93 & 514 & 2.00 & 0.60 \\
PSC & 53 & 35 & 45 & 15 & -0.47 & 0.53 & 0.80 & 39 & 58 & 0.65 & 0.71 \\
PPC & 53 & 18 & 35 & 11 & -0.61 & 0.42 & 0.68 & 27 & 49 & 0.56 & 0.52 \\
MVChla & 53 & 27 & 34 & 9 & -0.23 & 0.41 & 0.87 & 35 & 47 & 0.57 & 0.76 \\
DVChla & 53 & 32 & 99 & 65 & -0.57 & 0.74 & 0.83 & 40 & $1 . E+04$ & 1.45 & 0.56 \\
MVChlb & 53 & 79 & 220 & 164 & -1.49 & 1.48 & 0.74 & 90 & 321 & 1.79 & 0.65 \\
DVChlb & 53 & 97 & 255 & 199 & -5.71 & 1.85 & 0.28 & 100 & 382 & 2.32 & NA \\
Chlc $1 / 2$ & 53 & 31 & 38 & 11 & -0.48 & 0.45 & 0.87 & 39 & 53 & 0.63 & 0.75 \\
Chlc ${ }_{3}$ & 53 & 37 & 94 & 59 & -1.12 & 0.74 & 0.74 & 50 & 134 & 0.98 & 0.61 \\
But & 53 & 35 & 62 & 26 & -1.50 & 0.67 & 0.64 & 43 & 81 & 0.82 & 0.55 \\
Hex & 53 & 36 & 44 & 13 & -0.7 & 0.54 & 0.77 & 42 & 57 & 0.67 & 0.66 \\
Fuco & 53 & 36 & 89 & 53 & -0.96 & 0.77 & 0.76 & 44 & 149 & 1.01 & 0.64 \\
Peridin & 53 & 80 & 305 & 238 & -3.79 & 1.50 & 0.41 & 83 & 424 & 1.74 & 0.32 \\
Allo & 53 & 28 & 110 & 79 & -1.81 & 1.05 & 0.78 & 47 & 266 & 1.53 & $N A$ \\
Diadino & 53 & 31 & 619 & 581 & -2.22 & 0.97 & 0.42 & 38 & 1208 & 1.20 & 0.39 \\
Diato & 53 & 48 & 284 & 226 & -3.63 & 1.27 & 0.37 & 59 & 658 & 1.76 & 0.21 \\
Zea & 53 & 19 & 31 & 7 & -1.11 & 0.36 & 0.56 & 28 & 42 & 0.48 & 0.35 \\
Caro & 53 & 29 & 38 & 11 & -1.08 & 0.44 & 0.72 & 34 & 57 & 0.67 & 0.52 \\
Lutein & 53 & 80 & 117 & 71 & -6.27 & 1.19 & 0.26 & 87 & 245 & 1.56 & $N A$ \\
Viola & 53 & 71 & 191 & 131 & -2.55 & 1.22 & 0.61 & 78 & 314 & 1.55 & 0.44 \\
Neo & 53 & 47 & 71 & 33 & -1.13 & 0.82 & 0.85 & 64 & 94 & 1.02 & 0.77 \\
PE & 53 & 65 & 106 & 60 & -0.99 & 1.01 & 0.73 & 72 & 585 & 1.34 & 0.62 \\
\hline
\end{tabular}


b) Field data set hyperspectral resolution $380-700 \mathrm{~nm}$

\begin{tabular}{lcrrrrrrrrrr} 
Pigment & $N$ & $M D P D$ & $M P D$ & \multicolumn{1}{l}{$P B$} & $a$ & $R M S E$ & $R^{2}$ & $M D P D_{c V}$ & $M P D_{c V}$ & $R M S E_{c V}$ & $R_{c V}^{2}$ \\
\hline TChla & 53 & 30 & 38 & 10 & -0.22 & 0.40 & 0.84 & 32 & 43 & 0.49 & 0.77 \\
TChlb & 53 & 86 & 245 & 190 & -1.58 & 1.58 & 0.71 & 92 & 316 & 1.82 & 0.65 \\
PSC & 53 & 32 & 46 & 16 & -0.43 & 0.51 & 0.82 & 34 & 53 & 0.62 & 0.75 \\
PPC & 53 & 22 & 33 & 10 & 0.70 & 0.40 & 0.70 & 27 & 49 & 0.58 & 0.46 \\
MVChla & 53 & 29 & 39 & 10 & -0.28 & 0.45 & 0.84 & 34 & 44 & 0.50 & 0.79 \\
DVChla & 53 & 30 & 82 & 47 & -0.50 & 0.69 & 0.85 & 41 & 7289 & 1.35 & 0.59 \\
MVChlb & 53 & 78 & 218 & 163 & -1.43 & 1.45 & 0.75 & 88 & 285 & 1.66 & 0.69 \\
DVChlb & 53 & 100 & 243 & 188 & -6.22 & 1.93 & 0.22 & 108 & 370 & 2.41 & NA \\
Chlc $_{1 / 2}$ & 53 & 32 & 43 & 13 & -0.58 & 0.49 & 0.85 & 36 & 49 & 0.56 & 0.80 \\
Chlc & 53 & 34 & 94 & 59 & -1.16 & 0.76 & 0.73 & 42 & 130 & 0.92 & 0.66 \\
But & 53 & 44 & 58 & 22 & 0.68 & 0.63 & 0.68 & 50 & 102 & 0.91 & 0.49 \\
Hex & 53 & 31 & 45 & 15 & -0.59 & 0.49 & 0.80 & 36 & 53 & 0.60 & 0.70 \\
Fuco & 53 & 31 & 106 & 71 & -1.06 & 0.81 & 0.74 & 43 & 171 & 1.08 & 0.59 \\
Peridin & 53 & 82 & 298 & 232 & 0.40 & 1.51 & 0.40 & 85 & 534 & 1.78 & 0.33 \\
Allo & 53 & 33 & 86 & 54 & -1.63 & 1.00 & 0.80 & 40 & 197 & 1.33 & $N A$ \\
Diadino & 53 & 36 & 418 & 381 & -2.11 & 0.95 & 0.45 & 40 & 1061 & 1.19 & 0.39 \\
Diato & 53 & 56 & 295 & 231 & 0.34 & 1.30 & 0.34 & 60 & 663 & 1.77 & 0.18 \\
Zea & 53 & 20 & 32 & 8 & 0.52 & 0.37 & 0.52 & 29 & 45 & 0.52 & 0.26 \\
Caro & 53 & 31 & 42 & 13 & -1.24 & 0.47 & 0.68 & 33 & 50 & 0.54 & 0.62 \\
Lutein & 53 & 72 & 115 & 65 & 0.35 & 1.12 & 0.35 & 84 & 310 & 1.54 & NA \\
Viola & 53 & 70 & 175 & 116 & 0.62 & 1.20 & 0.62 & 74 & 248 & 1.42 & 0.51 \\
Neo & 53 & 46 & 71 & 33 & -1.12 & 0.82 & 0.85 & 65 & 100 & 1.05 & 0.76 \\
PE & 53 & 61 & 121 & 71 & -1.12 & 1.07 & 0.70 & 65 & 139 & 1.16 & 0.69 \\
\hline
\end{tabular}


c) Field data set MERIS band resolution

\begin{tabular}{lrrrrrrrrrrl} 
Pigment & $N$ & $M D P D$ & \multicolumn{1}{c}{$M P D$} & \multicolumn{1}{c}{$P B$} & $a$ & $R M S E$ & $R^{2}$ & $M D P D_{C V}$ & $M P D_{c V}$ & $R M S E_{c V}$ & $R_{c V}^{2}$ \\
\hline TChla & 53 & 21 & 33 & 8 & -0.23 & 0.41 & 0.83 & 28 & 42 & 0.54 & 0.72 \\
TChlb & 53 & 77 & 219 & 168 & -1.52 & 1.55 & 0.72 & 87 & 265 & 1.74 & 0.67 \\
PSC & 53 & 27 & 45 & 15 & -0.47 & 0.53 & 0.80 & 32 & 51 & 0.62 & 0.75 \\
PPC & 53 & 26 & 36 & 11 & -0.66 & 0.43 & 0.66 & 28 & 51 & 0.57 & 0.52 \\
MVChla & 53 & 26 & 36 & 10 & -0.28 & 0.45 & 0.84 & 31 & 45 & 0.54 & 0.77 \\
DVChla & 53 & 31 & 90 & 57 & -0.70 & 0.82 & 0.79 & 40 & 76569 & 1.50 & 0.54 \\
MVChlb & 53 & 86 & 205 & 148 & -1.49 & 1.48 & 0.74 & 90 & 251 & 1.63 & 0.71 \\
DVChlb & 53 & 110 & 226 & 167 & -5.78 & 1.86 & 0.27 & 100 & 1522 & 2.32 & NA \\
Chlc $1 / 2$ & 53 & 32 & 41 & 12 & -0.56 & 0.49 & 0.85 & 34 & 48 & 0.57 & 0.79 \\
Chlc ${ }_{3}$ & 53 & 30 & 90 & 53 & -1.18 & 0.76 & 0.72 & 39 & 115 & 0.89 & 0.69 \\
But & 53 & 37 & 65 & 29 & -1.60 & 0.69 & 0.62 & 44 & 95 & 0.90 & 0.50 \\
Hex & 53 & 28 & 44 & 14 & -0.68 & 0.53 & 0.77 & 37 & 57 & 0.69 & 0.64 \\
Fuco & 53 & 38 & 106 & 68 & -1.10 & 0.82 & 0.73 & 43 & 191 & 1.04 & 0.63 \\
Peridin & 53 & 66 & 287 & 222 & -3.79 & 1.50 & 0.41 & 85 & 438 & 1.83 & 0.32 \\
Allo & 53 & 36 & 118 & 83 & -2.06 & 1.12 & 0.75 & 46 & 1068190 & 1.52 & $N A$ \\
Diadino & 53 & 33 & 522 & 484 & -2.40 & 1.01 & 0.37 & 40 & 959 & 1.16 & 0.37 \\
Diato & 53 & 51 & 357 & 294 & -4.43 & 1.40 & 0.23 & 59 & 499 & 1.68 & 0.18 \\
Zea & 53 & 27 & 33 & 7 & -1.23 & 0.38 & 0.51 & 31 & 45 & 0.52 & 0.28 \\
Caro & 53 & 29 & 39 & 11 & -1.13 & 0.45 & 0.71 & 34 & 55 & 0.62 & 0.55 \\
Lutein & 53 & 72 & 123 & 75 & -5.81 & 1.15 & 0.31 & 84 & 270 & 1.38 & NA \\
Viola & 53 & 71 & 203 & 142 & -2.65 & 1.24 & 0.59 & 75 & 276 & 1.45 & 0.49 \\
Neo & 53 & 54 & 78 & 38 & -1.21 & 0.86 & 0.84 & 64 & 123 & 1.00 & 0.78 \\
PE & 53 & 56 & 117 & 69 & -1.09 & 1.05 & 0.70 & 67 & 156 & 1.28 & 0.65 \\
\hline
\end{tabular}


d) MERIS 1x1 matchup data set

\begin{tabular}{|c|c|c|c|c|c|c|c|c|c|c|c|}
\hline Pigment & $\mathrm{N}$ & $M D P D$ & $M P D$ & $P B$ & $a$ & RMSE & $R^{2}$ & $M D P D_{c v}$ & $M P D_{c v}$ & $R M S E_{c v}$ & $R_{c v}^{2}$ \\
\hline TChla & 139 & 29 & 37 & 10 & -0.49 & 0.54 & 0.72 & 32 & 47 & 0.64 & 0.67 \\
\hline TChlb & 139 & 77 & 1417 & 1325 & -3.97 & 2.14 & 0.26 & 80 & 4760 & 2.27 & 0.23 \\
\hline PSC & 139 & 42 & 65 & 29 & -0.74 & 0.71 & 0.74 & 42 & 59 & 0.79 & 0.70 \\
\hline PPC & 139 & 24 & 36 & 10 & -1.23 & 0.44 & 0.50 & 27 & 40 & 0.49 & 0.50 \\
\hline MVChla & 139 & 38 & 51 & 19 & -0.57 & 0.61 & 0.74 & 40 & 55 & 0.67 & 0.72 \\
\hline DVChla & 139 & 58 & 902 & 838 & -2.82 & 1.73 & 0.26 & 61 & 1141 & 1.90 & 0.19 \\
\hline MVChlb & 139 & 91 & 965 & 873 & -4.44 & 2.22 & 0.28 & 91 & 3. $E+06$ & 2.44 & 0.24 \\
\hline DVChlb & 139 & 129 & 204 & 151 & -7.30 & 1.80 & 0.09 & 123 & 262 & 2.06 & 0.05 \\
\hline Chlc $_{1 / 2}$ & 139 & 54 & 1039 & 977 & -2.73 & 1.47 & 0.41 & 56 & 1296 & 1.67 & 0.34 \\
\hline $\mathrm{Chlc}_{3}$ & 139 & 86 & 716 & 629 & -5.90 & 2.29 & 0.03 & 89 & 964 & 2.44 & NA \\
\hline But & 139 & 46 & 311 & 257 & -2.10 & 1.21 & 0.56 & 52 & 374 & 1.31 & 0.53 \\
\hline Hex & 139 & 44 & 584 & 534 & -1.82 & 1.22 & 0.50 & 51 & 602 & 1.25 & 0.50 \\
\hline Fuco & 139 & 41 & 954 & 901 & -2.49 & 1.41 & 0.47 & 47 & 1535 & 1.60 & 0.41 \\
\hline Peridin & 139 & 79 & 643 & 561 & -4.44 & 1.79 & 0.32 & 81 & 953 & 1.92 & 0.26 \\
\hline Allo & 139 & 77 & 126 & 88 & -6.66 & 1.42 & 0.21 & 78 & 168 & 1.56 & 0.14 \\
\hline Diadino & 139 & 54 & 266 & 217 & -2.30 & 1.14 & 0.50 & 55 & 300 & 1.26 & 0.46 \\
\hline Diato & 139 & 89 & 317 & 242 & -6.41 & 1.75 & 0.11 & 92 & 2. $E+05$ & 2.09 & NA \\
\hline Zea & 139 & 27 & 350 & 320 & -2.00 & 0.72 & 0.27 & 31 & 1210 & 0.86 & 0.21 \\
\hline Caro & 139 & 53 & 585 & 522 & -3.37 & 1.49 & 0.29 & 55 & 860 & 1.63 & 0.21 \\
\hline Lutein & 139 & 33 & 45 & 23 & -7.39 & 0.85 & 0.17 & 36 & 50 & 0.95 & 0.10 \\
\hline $\begin{array}{l}\text { Viola } \\
\text { no Neo } \\
\text { no PE }\end{array}$ & 139 & 95 & 185 & 125 & -6.72 & 1.51 & 0.14 & 97 & 226 & 1.66 & 0.07 \\
\hline
\end{tabular}


e) MERIS 3x3 matchup data set

\begin{tabular}{llrrrrrlrrrr} 
Pigment & $N$ & $M D P D$ & $M P D$ & \multicolumn{1}{c}{$P B$} & $a$ & $R M S E$ & $R^{2}$ & $M D P D_{c V}$ & $M P D_{c V}$ & $R M S E_{c V}$ & $R_{c V}^{2}$ \\
\hline TChla & 155 & 28 & 36 & 10 & -0.47 & 0.52 & 0.74 & 29 & 47 & 0.65 & 0.64 \\
TChlb & 155 & 75 & 1402 & 1314 & -3.81 & 2.07 & 0.28 & 77 & $1 . E+04$ & 2.18 & 0.25 \\
PSC & 155 & 36 & 67 & 32 & -0.76 & 0.72 & 0.73 & 38 & 72 & 0.83 & 0.67 \\
PPC & 155 & 24 & 35 & 10 & -1.23 & 0.44 & 0.50 & 27 & 48 & 0.52 & 0.49 \\
MVChla & 155 & 36 & 54 & 21 & -0.58 & 0.62 & 0.74 & 39 & 59 & 0.73 & 0.67 \\
DVChla & 155 & 52 & 1190 & 1126 & -3.19 & 1.77 & 0.15 & 59 & $1 . E+05$ & 2.17 & 0.08 \\
MVChlb & 155 & 89 & 1047 & 960 & -4.16 & 2.15 & 0.32 & 90 & $5 . E+05$ & 2.32 & 0.26 \\
DVChlb & 155 & 120 & 205 & 150 & -7.29 & 1.80 & 0.09 & 137 & 5214 & 2.10 & NA \\
Chlc $1 / 2$ & 155 & 53 & 957 & 894 & -2.75 & 1.46 & 0.41 & 56 & 1170 & 1.58 & 0.37 \\
Chlc 3 & 155 & 89 & 701 & 612 & -6.16 & 2.31 & 0.00 & 89 & 9704 & 2.44 & NA \\
But & 155 & 46 & 305 & 249 & -2.17 & 1.21 & 0.55 & 50 & 338 & 1.33 & 0.51 \\
Hex & 155 & 41 & 477 & 428 & -1.76 & 1.16 & 0.52 & 45 & 530 & 1.23 & 0.52 \\
Fuco & 155 & 44 & 1177 & 1122 & -2.61 & 1.45 & 0.45 & 49 & 1604 & 1.66 & 0.37 \\
Peridin & 155 & 78 & 609 & 529 & -4.24 & 1.74 & 0.35 & 81 & 766 & 1.84 & 0.31 \\
Allo & 155 & 78 & 149 & 107 & -6.34 & 1.46 & 0.24 & 78 & 281 & 1.59 & 0.18 \\
Diadino & 155 & 50 & 282 & 234 & -2.40 & 1.16 & 0.48 & 54 & 305 & 1.31 & 0.40 \\
Diato & 155 & 92 & 318 & 242 & -6.82 & 1.79 & 0.06 & 93 & $7 . \mathrm{E}+05$ & 2.09 & NA \\
Zea & 155 & 25 & 388 & 360 & -2.02 & 0.71 & 0.26 & 29 & 778 & 0.80 & 0.23 \\
Caro & 155 & 53 & 574 & 510 & -3.30 & 1.47 & 0.31 & 54 & 754 & 1.59 & 0.22 \\
Lutein & 155 & 36 & 45 & 23 & -6.72 & 0.83 & 0.24 & 38 & 50 & 0.91 & 0.16 \\
Viola & 155 & 186 & 198 & 132 & -7.47 & 1.57 & 0.05 & 138 & 336 & 1.75 & NA \\
no Neo & & & & & & & & & & & \\
no PE & & & & & & & & & & & \\
\hline
\end{tabular}


f) MERIS 5x5 matchup data set

\begin{tabular}{|c|c|c|c|c|c|c|c|c|c|c|c|}
\hline Pigment & $\mathrm{N}$ & $M D P D$ & $M P D$ & $P B$ & $a$ & RMSE & $R^{2}$ & $M D P D_{c v}$ & $M P D_{c v}$ & $R M S E_{c v}$ & $R_{c V}^{2}$ \\
\hline TChla & 160 & 27 & 36 & 10 & -0.47 & 0.52 & 0.74 & 28 & 48 & 0.66 & 0.62 \\
\hline TChlb & 160 & 75 & 1400 & 1313 & -3.83 & 2.06 & 0.28 & 77 & $6 . E+04$ & 2.14 & 0.26 \\
\hline PSC & 160 & 33 & 66 & 31 & -0.78 & 0.72 & 0.73 & 38 & 74 & 0.83 & 0.66 \\
\hline PPC & 160 & 22 & 34 & 10 & -1.21 & 0.43 & 0.51 & 24 & 44 & 0.50 & 0.48 \\
\hline MVChla & 160 & 35 & 53 & 21 & -0.59 & 0.62 & 0.74 & 38 & 58 & 0.74 & 0.66 \\
\hline DVChla & 160 & 54 & 1186 & 1120 & -3.19 & 1.75 & 0.14 & 58 & 1.E+05 & 2.08 & 0.09 \\
\hline MVChlb & 160 & 89 & 1030 & 941 & -4.28 & 2.16 & 0.30 & 90 & 2448 & 2.27 & 0.27 \\
\hline DVChlb & 160 & 137 & 211 & 156 & -7.39 & 1.79 & 0.08 & 145 & 3451 & 2.12 & NA \\
\hline $\mathrm{Chlc}_{1 / 2}$ & 160 & 52 & 916 & 854 & -2.79 & 1.44 & $\begin{array}{l}0.41 \\
<0.0\end{array}$ & 54 & 1088 & 1.56 & 0.36 \\
\hline $\mathrm{Chlc}_{3}$ & 160 & 90 & 681 & 592 & -6.20 & 2.31 & 1 & 90 & 3642 & 2.43 & NA \\
\hline But & 160 & 48 & 333 & 279 & -2.23 & 1.21 & 0.54 & 51 & 390 & 1.34 & 0.49 \\
\hline Hex & 160 & 42 & 488 & 438 & -1.81 & 1.20 & 0.51 & 45 & 530 & 1.25 & 0.52 \\
\hline Fuco & 160 & 45 & 1138 & 1082 & -2.67 & 1.46 & 0.45 & 50 & 1580 & 1.70 & 0.36 \\
\hline Peridin & 160 & 79 & 601 & 522 & -4.28 & 1.74 & 0.35 & 82 & 801 & 1.85 & 0.30 \\
\hline Allo & 160 & 77 & 146 & 104 & -6.39 & 1.45 & 0.23 & 79 & 376 & 1.57 & 0.19 \\
\hline Diadino & 160 & 45 & 282 & 234 & -2.40 & 1.15 & 0.48 & 52 & 312 & 1.31 & 0.40 \\
\hline Diato & 160 & 91 & 317 & 241 & -6.58 & 1.74 & 0.10 & 92 & $9 . E+05$ & 2.05 & $0.4 \mathrm{NA}$ \\
\hline Zea & 160 & 26 & 389 & 360 & -2.01 & 0.70 & 0.26 & 29 & 736 & 0.78 & 0.25 \\
\hline Caro & 160 & 50 & 567 & 505 & -3.28 & 1.47 & 0.31 & 53 & 832 & 1.60 & 0.23 \\
\hline Lutein & 160 & 35 & 44 & 22 & -6.77 & 0.83 & 0.23 & 36 & 48 & 0.93 & 0.16 \\
\hline $\begin{array}{l}\text { Viola } \\
\text { no Neo } \\
\text { no PE }\end{array}$ & 160 & 193 & 195 & 129 & -7.53 & 1.57 & 0.04 & 145 & 427 & 1.70 & NA \\
\hline
\end{tabular}

